\title{
Fatty Liver in COVID-19: A Risk Factor or a Common Receptor?
}

\author{
Amir Anushiravani, $\mathrm{MD}^{1}$; Amir Reza Radmard, $\mathrm{MD}^{2}$; Maryam Rayatpisheh, $\mathrm{MD}^{{ }^{*}}$ \\ ${ }^{1}$ Digestive Disease Research Institute, Shariati Hospital, Tehran University of Medical Sciences, Tehran, Iran \\ ${ }^{2}$ Department of Radiology, Shariati Hospital, Tehran University of Medical Sciences, Tehran, Iran
}

\section{Dear Editor,}

A recent outbreak of the novel coronavirus (SARSCOV-2) has been posing significant threats to global health since December 2019. It originated from Wuhan, China and was declared a pandemic by the World Health Organization (WHO) on March 11, 2020. There are 63087137 confirmed cases of COVID-19 worldwide as of November 30, 2020, causing 1465368 deaths (case fatality rate of $2.3 \%$ ) according to the WHO COVID-19 situation report. Even though the crisis started in China, the total number of confirmed cases have been surpassed by the United States and Italy, with mortalities the highest in Italy and Spain. There are over 948749 confirmed cases in Iran with 47874 deaths caused by COVID- 19 .

SARS-COV-2 has caused clusters of severe respiratory diseases, leading to ICU admissions and respiratory failure. Extra-pulmonary manifestations have been reported such as diarrhea and elevated liver enzymes. ${ }^{1}$ Liver diseases have been reported in $60 \%$ of patients with SARS $^{2}$ and since its genome sequence is more than $80 \%$ similar with the novel SARS-CoV-2, we should pay more attention to the largest organ in our body. The liver may be affected by viral exposure, hepatotoxic drugs, immunemediated inflammation, and possibly by similar receptors and pathways that facilitate COVID-19 entry into cells. Several case studies, all from China, have shown different clinical features of COVID-19, in which 2-11\% had hepatic comorbidities and abnormal aminotransferases were reported in $14 \%-53 \%$ of cases, ${ }^{3-5}$ where liver injury was more prevalent in severe cases.

To the best of our knowledge, non-alcoholic fatty liver disease (NAFLD) has not been mentioned in association with this novel viral disease. Here, we present our experience on patients suspicious for COVID-19 pneumonia who visited our emergency department in one of the main referral hospitals affiliated with Tehran University of Medical Sciences, Tehran, Iran from February 20 to March 28, and underwent chest CT scan. From a total of 441 patients who underwent CT scan in our outpatient setting, 125 (28.3\%) were highly suggestive for COVID-19 pneumonia. According to the density of liver on non-contrast CT scan for non-invasive diagnosis of steatosis, ${ }^{6}$ we noticed that moderate to severe fatty liver disease was significantly more prevalent in those with COVID-19 pneumonia ( $16.8 \%$ vs $2.2 \%, P<0.0001$ ), when liver was assessed in the upper abdomen cuts of chest CT scans (Figure 1). Patients in both groups were not significantly different in terms of their age $(52.66 \pm 4.25$ vs. $54.58 \pm 3.54$ ), gender (males were $46.98 \pm 2.21$ vs. $48.84 \pm 2.58)$, body mass index $(24.74 \pm 3.57$ vs. $24.22 \pm 3.52$ ), and risk factors such as diabetes mellitus type $2(9.50 \% \pm 1.23$ vs. $8.23 \% \pm 1.33)$, hypertension $(12.25 \pm 1.21$ vs $10.32 \pm 1.88)$, and cardiovascular diseases $(8.75 \pm 1.55$ vs $9.65 \pm 2.20)$.

NAFLD has a prevalence of 20\%-35\% in Iranian adults, mainly seen in those with overweight and obesity. ${ }^{7}$ Sanctions have forced us to limit CT scans to patients $<100$ $\mathrm{kg}$ in weight, in order to preserve our present equipment.

We know that human transmission and pathogenesis of COVID-19 depends on virus attachment, receptor recognition, protease cleavage and membrane fusion of the so-called S-protein, angiotensin converting enzyme 2 (ACE2), and the host transmembrane serine protease. ${ }^{8}$ Bulk RNA-seq data of paracarcinoma normal tissues were taken from The Cancer Genome Atlas (TCGA) and ACE2 mean expression was measured in different organs, with the liver showing an ACE2 mean expression of $6.86 \pm$ $1.351,{ }^{9}$ which was higher than the lungs $(5.83 \pm 0.710)$.

Recently, $\mathrm{Xu}$ et al reported a 50 -year-old man who had died of COVID-19, with post-mortem liver biopsies showing moderate microvesicular steatosis and mild lobular and portal activity. ${ }^{10}$

In a retrospective study performed by Medeiros et al on 316 patients (204 RT-PCR positive; 112 RT-PCR negative), regression analysis showed a 4.7-times odds of steatosis in the COVID-19 positive group as compared to controls (OR: 4.698; 95\% CI: 2.12-10.41, P<0.001). ${ }^{11}$

Whether NAFLD predisposes patients to COVID-19 or having common receptors and pathways causes their co-occurrence needs to be meticulously evaluated. The association between moderate to severe fatty liver disease and COVID-19 may lead to better understanding of the 


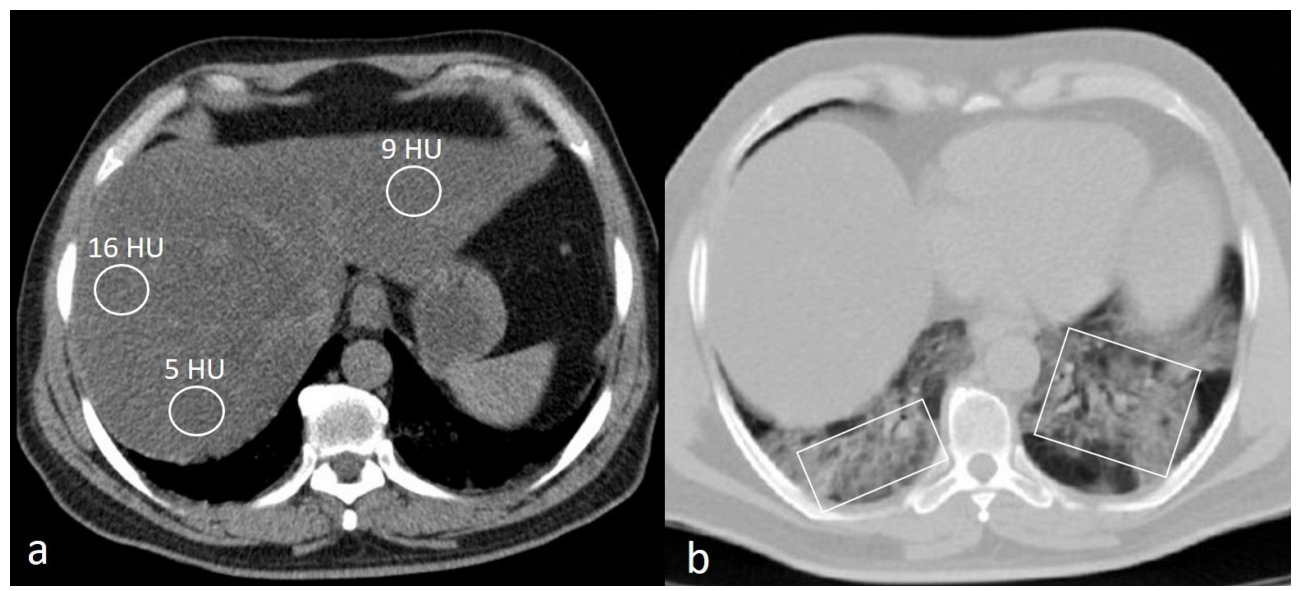

Figure 1. Non-contrast Chest CT Scan of a 52-Year-Old Male Patient with Fever, Cough and Myalgia Without Respiratory Distress Who Had an Unremarkable Past Medical History. (a) Mean density of regions of interest drawn at both hepatic lobes is 10 Hounsfield unit (HU) consistent with severe fatty liver. (b) There are bilateral patchy ground glass opacities and early consolidations (boxes) in lower lobes highly suggestive for COVID-19 pneumonia.

emerging, rapidly evolving pandemic.

\section{Authors' Contribution}

AA and ARR had an effective role in the implementation of this project and data collection. MR was involved in review of articles. AA and MR participated in writing the manuscript and analysis. ARR interpreted the imaging. All three authors have contributed effectively to the final arrangement of this letter.

\section{Conflict of Interest Disclosures}

The authors declare that they have no conflict of interest.

\section{Ethical Statement}

Not applicable.

\section{References}

1. Gu J, Han B, Wang J. COVID-19: gastrointestinal manifestations and potential fecal-oral transmission. Gastroenterology. 2020;158(6):1518-9. doi: 10.1053/j. gastro.2020.02.054.

2. Chau TN, Lee KC, Yao H, Tsang TY, Chow TC, Yeung YC, et al. SARS-associated viral hepatitis caused by a novel coronavirus: report of three cases. Hepatology. 2004;39(2):302-10. doi: 10.1002/hep.20111.

3. Huang C, Wang Y, Li X, Ren L, Zhao J, Hu Y, et al. Clinical features of patients infected with 2019 novel coronavirus in Wuhan, China. Lancet. 2020;395(10223):497-506. doi: 10.1016/s0140-6736(20)30183-5.

4. Guan WJ, Ni ZY, Hu Y, Liang WH, Ou CQ, He JX, et al. Clinical characteristics of coronavirus disease 2019 in
China. N Engl J Med. 2020;382(18):1708-20. doi: 10.1056/ NEJMoa2002032.

5. Zhang C, Shi L, Wang FS. Liver injury in COVID-19: management and challenges. Lancet Gastroenterol Hepatol. 2020;5(5):428-30. doi: 10.1016/s2468-1253(20)30057-1.

6. Pickhardt PJ, Park SH, Hahn L, Lee SG, Bae KT, Yu ES. Specificity of unenhanced CT for non-invasive diagnosis of hepatic steatosis: implications for the investigation of the natural history of incidental steatosis. Eur Radiol. 2012;22(5):1075-82. doi: 10.1007/s00330-011-2349-2.

7. Anushiravani A, Ghajarieh Sepanlou S. Burden of liver diseases: a review from Iran. Middle East J Dig Dis. 2019;11(4):189-91. doi: 10.15171/mejdd.2019.147.

8. Huang Q, Herrmann A. Fast assessment of human receptorbinding capability of 2019 novel coronavirus (2019-nCoV). bioRxiv. 2020. doi: 10.1101/2020.02.01.930537.

9. Xu H, Zhong L, Deng J, Peng J, Dan H, Zeng X, et al. High expression of ACE2 receptor of 2019-nCoV on the epithelial cells of oral mucosa. Int J Oral Sci. 2020;12(1):8. doi: 10.1038/s41368-020-0074-x.

10. Xu Z, Shi L, Wang Y, Zhang J, Huang L, Zhang C, et al. Pathological findings of COVID-19 associated with acute respiratory distress syndrome. Lancet Respir Med. 2020;8(4):420-2. doi: 10.1016/s2213-2600(20)30076-x.

11. Medeiros AK, Barbisan CC, Cruz IR, de Araújo EM, Libânio BB, Albuquerque KS, et al. Higher frequency of hepatic steatosis at CT among COVID-19-positive patients. Abdom Radiol (NY). 2020;45(9):2748-54. doi: 10.1007/ s00261-020-02648-7. 